\title{
Mechanical Behaviour of Nanostructured Iron Fabricated by Severe Plastic Deformation under Diffusion Flow of Nitrogen
}

\author{
A.I. Yurkova ${ }^{1, a}$, A.V. Byakova ${ }^{1 ; 2, b}$, A.V. Belots'ky ${ }^{1, a}$, Yu. V. Milman ${ }^{2, b}$, and S.N. Dub $b^{3, c}$ \\ ${ }^{1}$ Department of Metal Physics, National Technical University of Ukraine "Kiev Polytechnic \\ Institute", 37 Prospect Peremohy, 03056 Kiev, Ukraine \\ ${ }^{2}$ Phase Transformation Division, Institute for Problems of Material Science, National \\ Academy of Sciences of Ukraine, 3 Krzhyzhanivs'ky St., 03142, Kiev, Ukraine \\ ${ }^{3}$ Institute of Superhard Materials, National Academy of Sciences of Ukraine, 2 \\ Avtozavodskay St., 04074, Kiev, Ukraine \\ ae-mail: yurkova@list.ru, be-mail: byakova@vic.com.ua, ${ }^{\mathrm{c} e}$ e-mail:Iz@ism.kiev.ua
}

Keywords: severe plastic deformation, friction, nitriding, deformation structure, nano-grained structure, nanoindentation, Young`s modulus, hardness, plasticity characteristic $\delta_{A}$

\begin{abstract}
Specific features of mechanical behaviour of ultra fine grained iron subjected to friction treatment with nitriding (FN) were clarified by comparison with that induced by friction treatment (FT) with air. Mechanical parameters such as Young's modulus, nanohardness, and plasticity characteristic $\delta_{A}$ were found to be of high sensitive both to the scale of grain structure and to iron modification by nitrogen. Young's modulus tends to decrease and Hall-Petch low fails to describe correlation between grain structure and hardness for submicro-grained and nanocrystalline iron. Hall-Petch coefficient, $k_{y}$, decreases as grain size decreases within submicro-grained and, then, nano grained sections and it takes even negative value in nano grained section modified by nitrogen. Parameter $\delta_{A}$ is found to be dependent on combination of hardness and Young's modulus, resulting in its variation with decreasing the grain size. The presence of secondary nanocrystalline $\mathrm{Fe}_{4} \mathrm{~N}$ phase fundamentally changes mechanical behaviour of nanocrystalline iron, leading to strengthening the grain boundaries and triple junctions.
\end{abstract}

\section{Introduction}

Nano-crystalline materials with a large number of grain boundaries and triple junctions have been found exhibit interesting combination of physical and mechanical properties, making them of growing attention to researches employed in scientific and engineering applications. However, these properties are strongly dependent on the route employed for producing nc materials [1]. Compared to others routes severe plastic deformation (SPD) technique offers to essential advantages for ultra grain refining the structure of metallic materials, making them of $100 \%$ dense and free of contaminations. Among other properties mechanical parameters of nano-crystalline (nc) and sub micro-crystalline $(\mathrm{smc})$ materials are of great importance. These materials demonstrate specific features of mechanical behaviour [1-7]. However, results published in the literature are of controversial nature and their explanation is under dispute.

The present study aims to investigate the specific features of mechanical behaviour of iron subjected to severe plastic deformation under diffusion flow of dissolved dopant element (SPDD) and occurred due to friction with gas atmosphere, i. e. either with nitriding atmosphere or with air.

\section{Experimental}

Processing procedure. Samples of iron with purity 99.97 wt. \% and grain size about $100 \mu \mathrm{m}$ were processed either by friction treatment with ammonia gas (FN) or by friction treatment (FT) with air. Detailed description for FT and FN processes can be found [8]. In addition, iron sample obtained by FN process were further subjected to natural ageing during more than five years. 
Mechanical testing. To study scale effect on material response to deformation load displacement experiments were performed in cross-section of the sample by using Nano Indenter $\mathrm{II}^{\mathrm{R}}$ tester with Berkovich indenter. Mechanical parameters such as Young's modulus $E$, nanohardness $H$ and plasticity characteristics $\delta_{A}$ and $\delta_{D}$ were determined from complete cycle of loading and unloading data. Young's modulus was computed according to the method procedure of Oliver and Pharr [9]. Mechanical parameters were obtained in cross-section of treated samples. Two kinds of plasticity characteristics such as $\delta_{A}$ and $\delta_{D}$ of physical meaning nearly the same as plasticity characteristics $\delta_{H}$ [10] were derived using loading and unloading data, as shown in Fig.1. As applied for iron sample the values of these characteristics were found to be quite similar.

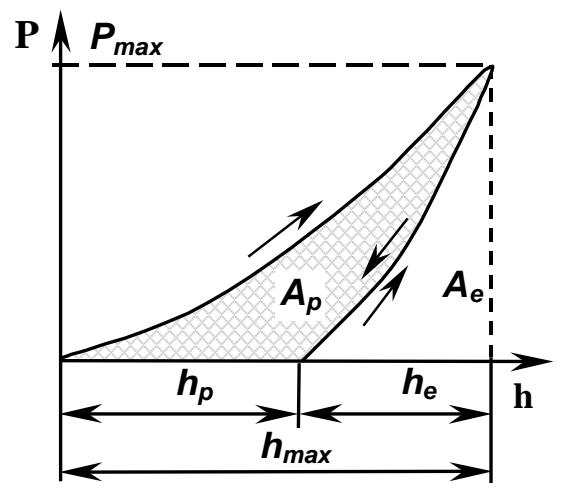

Fugure1: Diagram of load-displacement curves in the coordinates such as P-loading force and h- approaching of the indenter-specimen couple: $\delta_{A}=\frac{A_{p}}{A}$ is a share of plastic component $A_{p}$, which is defined by the area between loading and unloading data, in the total area under loading data $A=A_{e}$ $+\mathrm{A}_{\mathrm{p}}$ (where $\mathrm{A}_{\mathrm{e}}$ is elastic component); $\delta_{D}=\frac{h_{p}}{h_{\max }}$ is a share of plastic displacement, $h_{p}$, to the total displacement, $h_{\max }$.

\section{Results}

Structural characterisation. The results of XRD analysis and TEM observation [8] testified that FN and FT lead to formation of thin surface layers attributed to proper chemical compounds that are followed by structural sections of different scale regimes, as shown in Fig.2a.
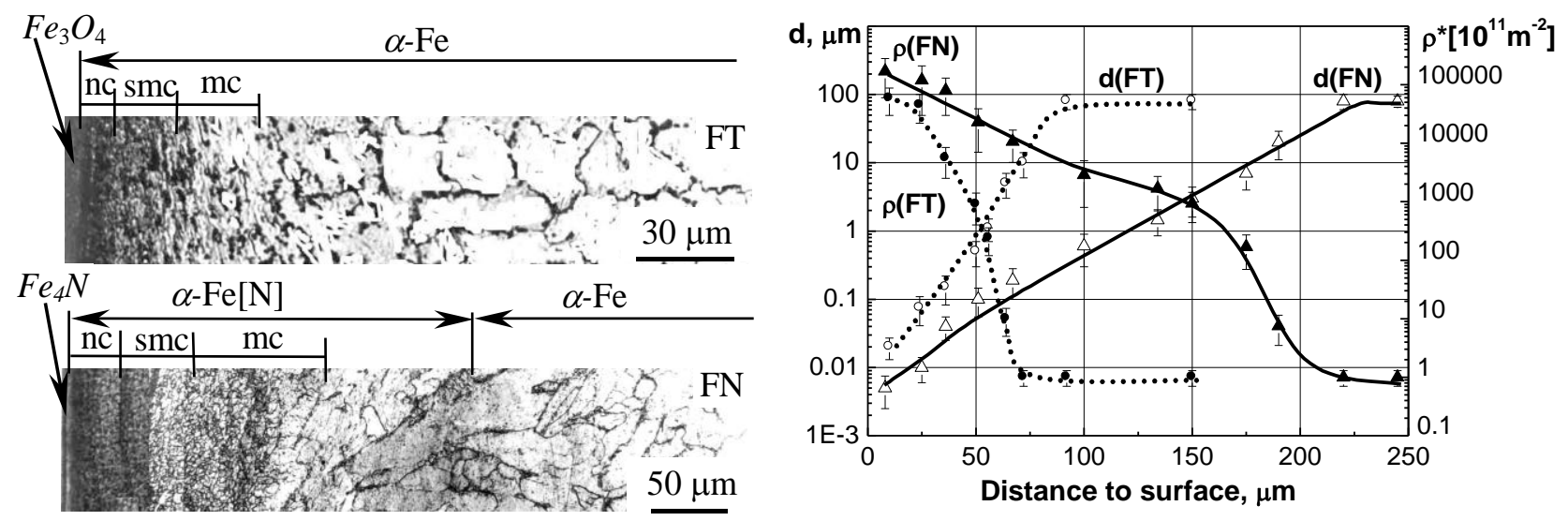

(a)

Figure 2. (a) Optical micrographs of deformation structures induced in iron by FT and FN as well as (b) variation of grain size and dislocation density along (a) structural sections attributed to nano-crystalline $(\mathrm{nc})$ regime, sub micro-crystalline $(\mathrm{smc})$ regime, and microcrystalline $(\mathrm{mc})$ regime.

Grain size plotted along the deformation-induced layer is shown in Fig.2b together with the data for dislocation density. The results indicate that extension of deformation layer induced by FN becomes larger and dislocation density reaches greater values compared to those subjected to FT. Moreover, finally stabilised nano-grain size induced by FN is nearly by 3 times smaller than that induced by FT [8]. It is notable that nitrogen content in ultra fine grained $\alpha-\mathrm{Fe}[\mathrm{N}]$ increases dramatically up to $0.3 \mathrm{wt} . \%$ at the greatest and the difference reaches $10^{2}$ times as compared to conventionally grained $\alpha-\mathrm{Fe}[\mathrm{N}]$ obtained by currently accepted furnace nitriding [8]. However, no 
indication to the presence of secondary nanocrystalline $\gamma^{\prime}$-phase $\left(\mathrm{Fe}_{4} \mathrm{~N}\right.$-nitride $)$ was obtained by TEM observation within deformation-induced layer of $\alpha$-Fe[N]. Secondary nanocrystalline $\gamma$ '-phase ( $\mathrm{Fe}_{4} \mathrm{~N}$-nitride) appeared within $\alpha-\mathrm{Fe}[\mathrm{N}]$ of nc-regime after natural ageing.

Mechanical properties of $\alpha$-Fe subjected to FT route. Fig. 3a shows the scale effect of grain structure on mechanical parameters of $\alpha$-Fe processed by FT route. Young's modulus tends to decrease as grain size decreases down to sub micro-metre scale and it reaches the smallest value, which is about $15 \%$ less than that for conventionally grained matrix, as could be seen in Fig. $3 a$.
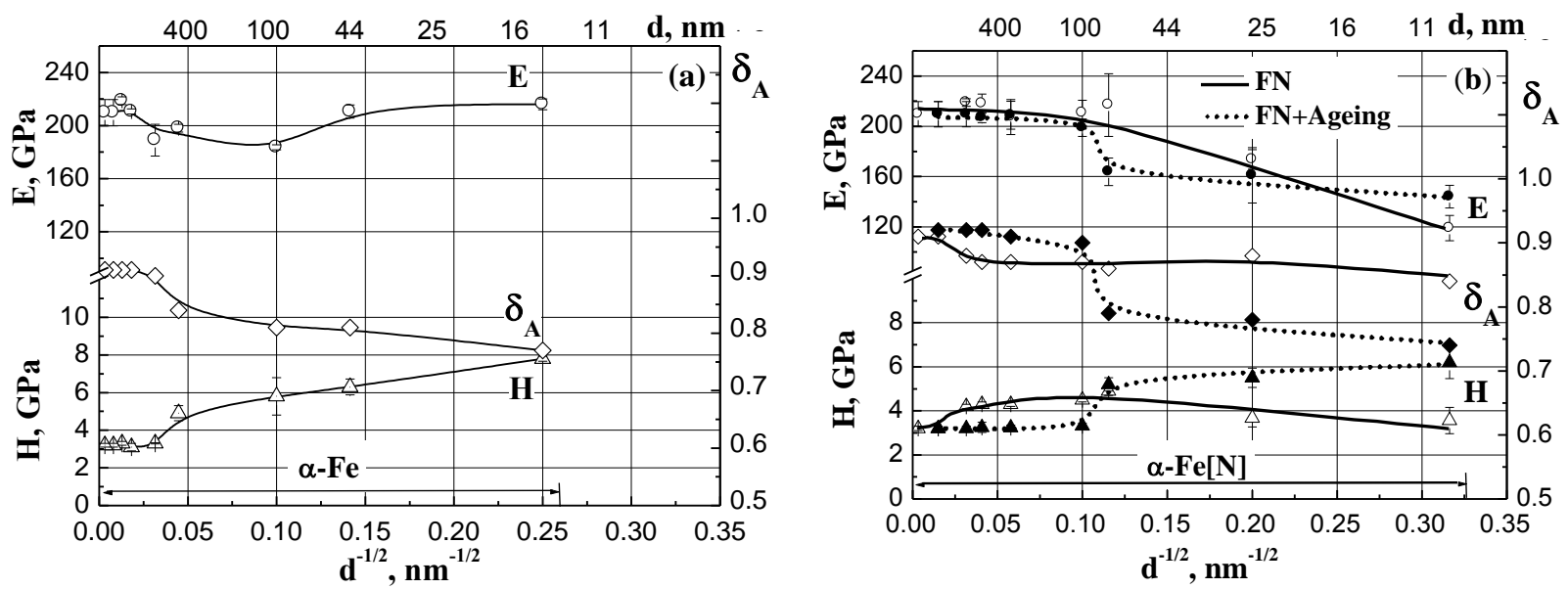

Figure 3. Relation between mechanical parameters and grain size $\mathrm{d}^{-1 / 2}$ : (a) FT; (b) FN, FN+ageing

However, Young's modulus steeply re-increases as grain size further decreases down to nanoscale level and it reaches nearly the same value as that for conventionally grained matrix. By comparison with the data listed in Table 1 it could be seen that the value of Young's modulus for smc $\alpha$-Fe is roughly by $25 \%$ higher than that for fine grained $\mathrm{Fe}_{3} \mathrm{O}_{4}$-oxide layer.

Table 1. Mechanical parameters for layer of chemical compounds

\begin{tabular}{|c|c|c|c|}
\hline Phase & Young's modulus $E[\mathrm{GPa}]$ & Nanohardness $H[\mathrm{GPa}]$ & Plasticity characteristic $\delta_{A}$ \\
\hline $\mathrm{Fe}_{3} \mathrm{O}_{4}$ & $144 \pm 3$ & $9.7 \pm 1.8$ & 0.63 \\
\hline $\mathrm{Fe}_{4} \mathrm{~N}$ & $172 \pm 12$ & $8.3 \pm 0.7$ & 0.65 \\
\hline
\end{tabular}

Fig. 3a shows that nanohardness of $\alpha$-Fe monotonically increases with decreasing the grain size from conventional value to that of nano-scaled level and it performs the highest value in the ncsection adjacent to $\mathrm{Fe}_{3} \mathrm{O}_{4}$-oxide layer. This variation of nanohardness is accompanied by the decrease of plasticity characteristic $\delta_{A}$.

Mechanical properties of $\alpha-\mathrm{Fe}[\mathrm{N}]$ subjected to $\mathrm{FN}$ route. Variation of mechanical parameters via grain size induced by FN is shown in Fig. 3b. It could be seen that the decrease of grain size down to nano-sized level causes Young's modulus for $\alpha$-Fe[N] to significant decrease, making them in nc-regime by $25 \%$ smaller than that for fine grained layer of $\mathrm{Fe}_{4} \mathrm{~N}$-nitride listed in Table 1 The difference between Young's modulus for nc $\alpha-\mathrm{Fe}[\mathrm{N}]$ enriched by nitrogen up to $0.3 \mathrm{wt} . \%$ and that for conventionally grained $\alpha-\mathrm{Fe}[\mathrm{N}]$ modified by $0.003 \mathrm{wt} . \%$ of nitrogen reaches about $50 \%$. In addition, Young's modulus for mc $\alpha-\mathrm{Fe}[\mathrm{N}]$ modified by $0.003 \mathrm{wt} . \%$ nitrogen remains the same as that for $\alpha$-Fe matrix. It is notable that Young's modulus for smc $\alpha-\mathrm{Fe}[\mathrm{N}]$ is much superior to that for $\alpha-\mathrm{Fe}$ of the same structural regime whereas the opposite is true for nc $\alpha-\mathrm{Fe}[\mathrm{N}]$ for which Young's modulus is much smaller than that for nc $\alpha$-Fe subjected to FT.

Fig. $3 \mathrm{~b}$ shows that nanohardness of $\alpha-\mathrm{Fe}[\mathrm{N}]$ increases as grain size decreases in region from conventional value to that of sub micro-metre scale and, thus, it reaches the largest value at the critical grain size of about $100 \mathrm{~nm}$. Further decreasing the grain size causes nanohardness of 
$\alpha-\mathrm{Fe}[\mathrm{N}]$ to decrease gradually. It is notable that nanohardness detected in nc $\alpha-\mathrm{Fe}[\mathrm{N}]$ adjacent to $\mathrm{Fe}_{4} \mathrm{~N}$-nitride layer reaches nearly the same value as that for conventionally grained matrix and it is by two times smaller than that for $\mathrm{Fe}_{4} \mathrm{~N}$-nitride layer listed in Table 1. By comparison the data presented in Figs. $3 \mathrm{a}$ and $3 \mathrm{~b}$ it could be seen that nanohardness of nc $\alpha$-Fe[N] demonstrates the values, which is about two times smaller than those for nc $\alpha$-Fe subjected to FT.

Despite of varying nanohardness via deformation-induced layer, plasticity characteristic $\delta_{A}$ remains almost unchanged with decreasing the grain size down to nano-metre scale, as shown in Fig. 3b. Generally, the value of parameter $\delta_{A}$ for $\mathrm{nc} \alpha-\mathrm{Fe}[\mathrm{N}]$ is superior by $25 \%$ to that for $\mathrm{Fe}_{4} \mathrm{~N}$-layer listed in Table 1 .

Mechanical properties of $\alpha-F e[N]$ induced by $F N$ and subjected to further natural ageing. The data shown in Fig $3 b$ indicate that further natural ageing the iron processed by FN causes the values of Young's modulus for $\mathrm{nc} \alpha-\mathrm{Fe}[\mathrm{N}]$ to increase. Moreover, natural ageing causes correlation of nanohardness $v s$. grain size within $\mathrm{nc} \alpha-\mathrm{Fe}[\mathrm{N}]$ to inversion. Therefore, nanohardness of $\alpha-\mathrm{Fe}[\mathrm{N}]$ after natural ageing increases continually as grain size decreases in region from conventional value to that of nano-metre scale. As a result of this effect one can see that nanohardness for nc $\alpha$-Fe[N] after further ageing becomes higher than that for initially obtained $\mathrm{nc} \alpha-\mathrm{Fe}[\mathrm{N}]$ whereas the opposite is true for smc $\alpha-\mathrm{Fe}[\mathrm{N}]$ for which further ageing ensures nanohardness values the same as those for conventionally grained matrix. As compared with initially obtained data attributed to FN, plasticity characteristic $\delta_{A}$ of nc $\alpha-\mathrm{Fe}[\mathrm{N}]$ after ageing becomes smaller. Unlike the effect above plasticity characteristic $\delta_{A}$ of smc $\alpha-\mathrm{Fe}[\mathrm{N}]$ after ageing increases and it reaches the values nearly the same as those for conventionally grained matrix.

\section{Discussion}

The results of the present study show appreciable effect of ultra fine refining the grain structure on material response to deformation. In general, mechanical behaviour of material could be influenced by several structural parameters, i.e. dissolved dopant elements, dislocations and other stacking faults, grain boundaries, secondary phase. As applied to fine grained deformation structure induced by SPD, the contribution of these factors is yet open to question.

Young's modulus of $\alpha$-Fe obtained by FT tends to be smaller with reducing the grain size down to sub micro-metre scale, as shown in Fig.3a. The scale effect on Young's modulus is revealed too for $\mathrm{nc} \alpha-\mathrm{Fe}[\mathrm{N}]$ induced by FN, as evidenced in Fig. $3 \mathrm{~b}$.

Several mechanisms were suggested in the literature for the effect of the grain size on elasticity properties of materials fabricated by SPD technique. It was assumed that the decrease of elastic properties for nc materials is caused by imperfections, i.e. microstrains within the nano-grains that occurred due to dislocations and stacking faults $[5,6]$. According to "composite model" of Mughrabi [11] the former effect could be resulted from the large number of grain boundaries and, thus, increased volume fraction of the triple junctions for which mechanical properties are different from those of grain interior. That is why distorted elasticity of grain boundaries and triple junctions are considered to be capable for decreasing elasticity modulus of nc material $[2,5,6]$.

As notable characteristic, one can notice that further natural ageing mainly changes the relation between the grain size and Young's modulus of $\mathrm{nc} \alpha-\mathrm{Fe}[\mathrm{N}]$, as shown in Fig. 3b. Young's modulus for $\mathrm{nc} \alpha-\mathrm{Fe}[\mathrm{N}]$ increases when grain size is limited to less than $20 \mathrm{~nm}$ whereas the opposite is true for $\alpha-\mathrm{Fe}[\mathrm{N}]$ refined to larger grain size. The increases of Young's modulus could be caused by the appearance of secondary nanocrystalline $\mathrm{Fe}_{4} \mathrm{~N}$-phase, leading to strengthening the grain boundaries/triple junctions and, thus, to correction of their distorted elasticity. Following the mentioned above re-increase of Young's modulus for $\alpha$-Fe occurred as the grain size further decreases down to nanometre scale, as shown in Fig. 3a, could be attributed to the effect of nanocrystalline $\mathrm{Fe}_{3} \mathrm{O}_{4}$-oxides. The presence of oxide precipitation $\mathrm{Fe}_{3} \mathrm{O}_{4}$-phase in nc $\alpha$ - $\mathrm{Fe}$ is thought to be realistic by considering the experimental data [12], which showed by TEM 
observation that formation of nano-scaled $\mathrm{Cr}_{2} \mathrm{O}_{3}$-oxide at grain boundaries/triple junctions of nc chromium coating can occur even under vacuum conditions during magnetron sputtering.

Attention is drawn to the fact that precipitation $\mathrm{Fe}_{4} \mathrm{~N}$-phase formed after ageing initiates redistribution of dissolved nitrogen within deformation-induced structure, leading to the reasonable decrease of nitrogen content in $\alpha-\mathrm{Fe}[\mathrm{N}]$. The former effect is thought to be responsible for decreasing the Young's modulus of smc $\alpha-\mathrm{Fe}[\mathrm{N}]$. This suggestion is confirmed by comparison the data for $\alpha-\mathrm{Fe}[\mathrm{N}]$ after FN to those for $\alpha$-Fe subjected to FT. It could be seen in Figs. 3a and 3b that the values of Young's modulus for smc $\alpha$ - $\mathrm{Fe}$ is smaller than those for $\alpha-\mathrm{Fe}[\mathrm{N}]$ of the same regime.

Nanohardness of ultra fine grained iron is found to be in actual conflict with Hall-Petch relation $\sigma_{\tau}=\sigma_{0}+k_{y} d^{-1 / 2}$, where $\sigma_{0}$ is the mean flow stress of single crystal, $k_{y}$ is a Hall-Petch coefficient, $d$ is grain size. Relation between stress and hardness is given by Tabor's concept $H=3 \sigma$.

The data listed in Table 2 show that $k_{y}{ }^{*}$ coefficient for ultra fine grain regimes is much smaller compared to the value $k_{y}=0.39 \mathrm{MPa} \cdot \mathrm{m}^{1 / 2}$ that is typical for conventionally grained iron. Furthermore, $k_{y}{ }^{*}$ coefficient tend to decrease as the grain size decreases in the range from smcregime to nc-regime. As applied to structural regime of the same scale $k_{y}{ }^{*}$ coefficient for $\alpha$-Fe[N] is by order magnitude is smaller than that for $\alpha$-Fe and it takes even negative value for $\alpha-\mathrm{Fe}[\mathrm{N}]$ of nc-regime. The results of the present study agree with the data related to the scale effect on hardening the ultra fine metallic structures induced by SPD and reported in [2, 5, 13]. Following "composite model" of Mughrabi [11] this abnormal mechanical behaviour one tries to explain by highly increased role of interfacial defects, leading to great allowance for grain boundaries sliding and even to grain rotation. However, the results presented in Table 2 indicate that secondary precipitation $\mathrm{Fe}_{4} \mathrm{~N}$-phase formed after further natural ageing fundamentally changes mechanical behaviour of ultra fine grained $\alpha$-Fe[N]. Appearance of precipitation $\mathrm{Fe}_{4} \mathrm{~N}$-phase causes $k_{y}{ }^{*}$ coefficient to increase, returning the positive value to this coefficient in nc-regime.

Table 2. Hall-Petch coefficient $k_{y}$ and $H_{0}$ value for single crystals of particular dislocation density

\begin{tabular}{|c|c|c|c|c|c|c|}
\hline Parameter & \multicolumn{2}{|c|}{$\alpha$-Fe } & \multicolumn{2}{c|}{$\alpha$-Fe[N] } & \multicolumn{2}{c|}{$\alpha$-Fe[N] after ageing } \\
\hline & nc-regime & smc-regime & nc-regime & smc-regime & nc-regime & smc-regime \\
\hline$H_{o}{ }^{*},[\mathrm{GPa}]$ & 4.49 & 4.18 & 5.53 & 4.12 & 4.85 & 3.20 \\
\hline$k_{y}{ }^{*},\left[\mathrm{MPa} \cdot \mathrm{m}^{1 / 2}\right]$ & 0.135 & 0.168 & -0.070 & 0.035 & 0.045 & 0.105 \\
\hline
\end{tabular}

It could be seen in Table 2 that the values of nanohardness $H_{0}{ }^{*}$ for ultra fine grained regimes induced by FT, FN, and FN with further ageing are different from that for conventionally grained iron, $H_{0}=3.0 \mathrm{GPa}$. The last one was determined in experiments with coarse-grained matrix for which size of grain about $100 \mu \mathrm{m}$ is much superior to that of nanohardness indentation. This evidence above allows us to suggest the appreciable contribution of dislocation density in nanohardness of ultra fine-grained structure induced by SPDD.

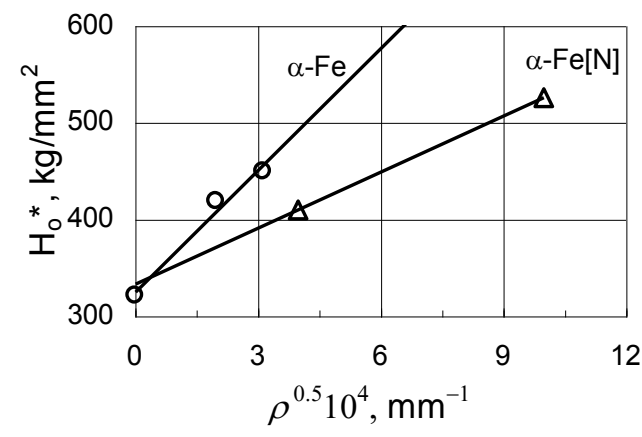

Figure 4. Relation between dislocation density, $\rho$, and nanohardness for $\alpha-\mathrm{Fe}$ and that for $\alpha-\mathrm{Fe}[\mathrm{N}]$.

For the data plotted in Fig. 4 hardening due to enhancing the dislocation density, $\rho$, occurred with reducing the grain size down to nano metre scale is satisfactory described by well known relation $\sigma_{\tau}=\sigma_{0}+\alpha G b \rho^{1 / 2}$ where $\sigma_{\tau}=\sigma_{o}{ }^{*}$ is the mean flow stress for single crystal of particular 
dislocation density, $\sigma_{0}$ is the mean flow stress for single crystal with no dislocations, $G$ is shear modulus, $b$ is the Burgers vector of dislocation in iron, $\alpha \approx 0.5$ is nondimensional coefficient.

Generally, the value of member $\alpha G b$ for refined $\alpha$-Fe is found to be the same as that calculated for iron when $G=82 \mathrm{GPa}$ and it is superior by order magnitude to that demonstrated by deformation-induced structure of $\alpha-\mathrm{Fe}[\mathrm{N}]$. From the physical point of view this fact indicates that solute nitrogen slows down the role of dislocation density in hardening ultra fine grained $\alpha-\mathrm{Fe}[\mathrm{N}]$.

Plasticity characteristic $\delta_{A}$. The results indicate that both nanohardness and Young's modulus contribute to variation of plasticity characteristic $\delta_{A}$, i.e. parameter $\delta_{A}$ increases as nanohardness decreases whereas the opposite is true when Young's modulus decreases.

Generally, $\mathrm{nc} \alpha$-Fe[N] after ageing demonstrates the greatest value of hardness and has plasticity characteristic $\delta_{A}$ that is only $15 \%$ smaller than critical value $\delta_{A} \approx 0.9$ at which bulk metallic materials exhibit brittle behaviour under uniaxial tensile tests [10].

\section{Concluding Remarks}

The results of the present study hold the key for greater understanding the relationships between elastic/plastic properties and multiphase deformation-induced structure of metallic materials including those reinforced by nanocrystalline particles of precipitation phase. Special attention should be paid to the development of novel modified technique denoted by SPDD that give the great allowance for improvement of nitriding process. In this way nanocrystalline metals and alloys treated by SPDD is believed to be promising both for the current basic research and for application in engineering practice.

\section{Acknowledgements}

This research is partly supported by Ministry of Education and Science of Ukraine, project \# 2730, and National Academy of Science of Ukraine, project \# N-4304.

\section{References}

[1] Nanostructured Materials: Scince and Technology. (Dordrecht: Kluwer Acad. Publ., 1998).

[2] R.A. Andrievski and A.M. Glezer: Scripta Metall. Vol. 44 (2001), \# 8/9, p. 1624.

[3] J.R.Weertman: Mateer. Sci. Eng., Vol. A166 (1993), p. 161.

[4] K.Lu , M.I.Sui : Scripta Met. Mater. Vol. 28 (1993), p. 1465.

[5] D.Korn, A.Morsch, R.Birringer, et al: J. Phys. Coll. C5. Suppl. Vol. 49 (1988), \# 10, p. 769.

[6] G.Palumbo, U.Erb, K.T.Aust: Scripta Met. Mater. Vol. 24 (1990), p. 2347.

[7] R.Z.Valiev, R.K.Islamgaliev, and I.V.Alexandrov: Progr.Mater.Sci. Vol. 45 (2000), p. 103.

[8] A.Yurkova, A.Belots`ky, A.Byakova. In: «Nanostructured Materials by High-Pressure Severe Plastic Deformation» (Kluver Acad. Publ., 2005, in press).

[9] W.C. Oliver, G.M. Pharr: J. Mater. Res. Vol. 7 (1992), p. 1564.

[10] Yu.V.Milman, B.A.Galanov, S.I.Chugunova: Acta. Metall. Mater. Vol. 41 (1993), \# 9, p. 2523

[11] H. Mughrabi: Acta metal. Vol. 31 (1983), p.1367.

[12] S.A.Firstov, T.G.Rogul, S.N.Dub, and et.al: Problem of materials Sci. Vol.1 (2002), 29, p. 202.

[13] C.S.Paude, R.A.Masumura, R.W. Amstrong: Nanostructured mater. Vol. 2 (1993), \#3, p. 323. 